\title{
On the structure of the directions not determined by a large affine point set
}

\author{
Jan De Beule, Peter Sziklai†, and Marcella Takáts
}

January 12, 2011

\begin{abstract}
Given a point set $U$ in an $n$-dimensional affine space of size $q^{n-1}-\varepsilon$, we obtain information on the structure of the set of directions that are not determined by $U$. We describe some applications in the theory of partial ovoids of certain generalized quadrangles.
\end{abstract}

\section{Introduction}

Let $\mathrm{PG}(n, q)$ and $\operatorname{AG}(n, q)$ denote the projective and the affine $n$-dimensional space over the finite field $\operatorname{GF}(q)$ of $q$ elements. Given a point set $U \subset \operatorname{AG}(n, q) \subset$ $\mathrm{PG}(n, q)$, a direction, i.e. a point $d \in H_{\infty}=\mathrm{PG}(n, q) \backslash \mathrm{AG}(n, q)$ is determined by $U$ if there is an affine line through $d$ which contains at least 2 points of $U$. Note that if $|U|>q^{n-1}$ then every direction is determined.

Many results on extendability of affine point sets not determining a given set of directions are known. The strongest results are known in the planar case. We mention the following theorem from [5].

Theorem 1 Let $U \subseteq \operatorname{AG}(2, q)$ be a set of affine points of size $q-k>q-\sqrt{q} / 2$, which does not determine a set $D$ of more than $(q+1) / 2$ directions. Then $U$ can be extended to a set of size $q$, not determining the set $D$ of directions.

An extendability result known for general dimension is the following. Originally, it was proved in [2] for $n=3$. A proof for general $n$ can be found in [1].

th:DBG_Ball Theorem 2 Let $q=p^{h}, p$ an odd prime and $h>1$, and let $U \subseteq \operatorname{AG}(n, q)$, $n \geq 3$, be a set of affine points of size $q^{n-1}-2$, which does not determine a set $D$ of at least $p+2$ directions. Then $U$ can be extended to a set of size $q$, not determining the set $D$ of directions.

The natural question is whether Theorem 2 can be improved in the sense that extendability of sets of size $q^{n-1}-\varepsilon$ is investigated, for $\varepsilon>2$, possibly with stronger assumptions on the number of non-determined directions.

\footnotetext{
*This author is a Postdoctoral Fellow of the Research Foundation Flanders - Belgium (FWO-Vlaanderen)

${ }^{\dagger}$ This research was partially supported by ... and OTKA T-49662,67867 grants.
} 
In this paper, we investigate affine point sets of size $q^{n-1}-\varepsilon$, for arbitrary $\varepsilon$, and where a non-trivial result is obtained when $\varepsilon$ is small. Instead of formulating an extendability result in terms of the number of non-determined directions, we formulate it in terms of the structure of the set of non-determined directions. We will show that a pointset $|U|=q^{n-1}-\varepsilon$ is always extendable to the size $q^{n-1}$, which we consider as the typical situation, or the set of non-determined directions is contained in an algebraic hypersurface of degree at most $\varepsilon(\varepsilon-$ $1)^{n-1}$ in the hyperplane at infinity of $\operatorname{AG}(n, q)$. Finally, we add a section with applications of the obtained theorem.

\section{The main result}

A point of $\operatorname{PG}(n, q)$ is represented by a homogenous $(n+1)$-tuple $\left(a_{0}, a_{1}, \ldots, a_{n}\right) \neq$ $(0,0, \ldots, 0)$. A hyperplane is the set of points whose coordinates satisfy a linear equation

$$
a_{0} X_{0}+a_{1} X_{1}+\ldots+a_{n} X_{n}=0
$$

and so hyperplanes are represented by a homogenous $(n+1)$-tuple $\left[a_{0}, a_{1}, \ldots, a_{n}\right] \neq$ $[0,0, \ldots, 0]$. Embed the affine space $\operatorname{AG}(n, q)$ in $\operatorname{PG}(n, q)$ such that the hyperplane $X_{0}=0$, i.e. the hyperplane with coordinates $[1,0, \ldots, 0]$ is the hyperplane at infinity of $\operatorname{AG}(n, q)$. Then the points of $\operatorname{AG}(n, q)$ will be coordinatized as $\left(1, a_{1}, a_{2}, \ldots, a_{n}\right)$.

The map $\delta$ from the points of $\operatorname{PG}(n, q)$ to its hyperplanes, mapping a point $\left(a_{0}, a_{1}, a_{2}, \ldots, a_{n}\right)$ to a hyperplane $\left[a_{0}, a_{1}, \ldots, a_{n}\right]$ is the standard duality of $\mathrm{PG}(n, q)$.

Let $U \subseteq \operatorname{AG}(n, q)$ be an affine point set, $|U|=q^{n-1}-\varepsilon$. Embed $\operatorname{AG}(n, q)$ in $\operatorname{PG}(n, q)$ and denote the hyperplane at infinity as $H_{\infty}$. Let $D \subseteq H_{\infty}$ be the set of directions determined by $U$ and put $N=H_{\infty} \backslash D$ the set of non-determined directions.

contnum Lemma 3 Let $0 \leq r \leq n-2$. Let $\alpha=\left(0, \alpha_{1}, \alpha_{2}, \alpha_{3}, \ldots, \alpha_{n}\right) \in N$ be a nondetermined direction. Then each of the affine subspaces of dimension $r+1$ through $\alpha$ contain at most $q^{r}$ points of $U$.

Proof. We prove it by the pigeon hole principle. An affine subspace of dimension $r+1$ through $\alpha$ contains $q^{r}$ affine (disjoint) lines through $\alpha$, and each line contains at most one point of $U$ as $\alpha$ is a non-determined direction.

Definition 4 If an affine subspace of dimension $r+1 \leq n-1$ through $\alpha \in N$ contains less than $q^{r}$ points of $U$, then it is called a deficient subspace. If it contains $q^{r}-t$ points of $U$, then its deficiency is $t$.

epsdefsub Corollary 5 Let $T \subseteq H_{\infty}$ be a subspace of dimension $r \leq n-2$ containing $\alpha \in N$. Then there are precisely $\varepsilon$ deficient subspaces of dimension $r+1$ (counted possibly with multiplicity) through $T$ (a subspace with deficiencyt is counted with multiplicity $t$ ).

In particular: 
epsline Corollary 6 There are precisely $\varepsilon$ affine lines through $\alpha$ not containing any point of $U$ (and $q^{n-1}-\varepsilon$ lines with 1 point of $U$ each).

Now consider the set $U=\left\{\left(1, a_{1}^{i}, a_{2}^{i}, a_{3}^{i}, \ldots, a_{n}^{i}\right): i=1, \ldots, q^{n-1}-\varepsilon\right\}$. We define its Rédei polynomial as follows:

$$
R\left(X_{0}, X_{1}, X_{2}, \ldots, X_{n}\right)=\prod_{i=1}^{q^{n-1}-\varepsilon}\left(X_{0}+a_{1}^{i} X_{1}+a_{2}^{i} X_{2}+\ldots+a_{n}^{i} X_{n}\right)
$$

The intersection properties of of the set $U$ with hyperplanes of $\mathrm{PG}(n, q)$ are translated into algebraic properties of the polynomial $R$ as follows. Consider $x_{1}, x_{2}, \ldots, x_{n} \in \mathrm{GF}(q)$, then $x \in \mathrm{GF}(q)$ is a root with multiplicity $m$ of the equation $R\left(X_{0}, x_{1}, x_{2}, \ldots, x_{n}\right)=0$ if and only if the hyperplane $\left[x, x_{1}, x_{2}, \ldots, x_{n}\right]$ contains $m$ points of $U$.

Define the set $S\left(X_{1}, X_{2}, \ldots, X_{n}\right)=\left\{a_{1}^{i} X_{1}+a_{2}^{i} X_{2}+\ldots+a_{n}^{i} X_{n}: i=1, \ldots, q^{n-1}-\right.$ $\varepsilon\}$, then $R$ can be written as

$$
R\left(X_{0}, X_{1}, X_{2}, \ldots, X_{n}\right)=\sum_{j=0}^{q^{n-1}-\varepsilon} \sigma_{q^{n-1}-\varepsilon-j}\left(X_{1}, X_{2}, \ldots, X_{n}\right) X_{0}^{j},
$$

where $\sigma_{j}\left(X_{1}, X_{2}, \ldots, X_{n}\right)$ is the $j$-th elementary symmetric polynomial of the set $S\left(X_{1}, X_{2}, \ldots, X_{n}\right)$.

Consider the subspace $s_{x_{1}, x_{2}, \ldots, x_{n}} \subset H_{\infty}=[1,0, \ldots, 0]$ of dimension $n-2$ which is the intersection of the hyperplanes $\left[x_{0}, x_{1}, x_{2}, \ldots, x_{n}\right], x_{0} \in \mathrm{GF}(q)$. Suppose that $s_{x_{1}, x_{2}, \ldots, x_{n}}$ contains an undetermined direction then, by Lemma 3, each of the hyperplanes different from $H_{\infty}$ through $s_{x_{1}, x_{2}, \ldots, x_{n}}$, contains at most $q^{n-2}$ points of $U$. This implies that there are precisely $\varepsilon$ such hyperplanes (counted with multiplicity) through $s_{x_{1}, x_{2}, \ldots, x_{n}}$ containing less than $q^{n-2}$ points of $U$ (a hyperplane with deficiency $t$ is counted with multiplicity $t$ ). Algebraically, this means that for the $(n-2)$-dimensional subspace $s_{x_{1}, x_{2}, \ldots, x_{n}}$,

$$
R\left(X_{0}, x_{1}, x_{2}, \ldots, x_{n}\right) f\left(X_{0}\right)=\left(X_{0}^{q}-X_{0}\right)^{q^{n-2}}
$$

where $f\left(X_{0}\right)=X_{0}^{\varepsilon}+\sum_{k=1}^{\varepsilon} f_{k} X_{0}^{\varepsilon-k}$ is a fully reducible polynomial of degree $\varepsilon$. Comparing the two sides of equation (1), one gets linear equations for the coefficients $f_{k}$ of $f$ in terms of the $\sigma_{j}\left(x_{1}, \ldots, x_{n}\right)$, and it is easy to see that the solutions for each $f_{k}$ are a polynomial expression in terms of the $\sigma_{j}\left(x_{1}, \ldots, x_{n}\right)$, $j=1, \ldots, k$, use e.g. Cramer's rule to solve the system of equations, and notice that the determinant in the denominator equals 1 . The polynomial expression is independent from the elements $x_{1}, x_{2}, \ldots, x_{n}$ (still under the assumption that $s_{x_{1}, x_{2}, \ldots, x_{n}}$ does contain an undetermined direction), so we can change them for the variables $X_{1}, X_{2}, \ldots, X_{n}$ which makes the coefficients $f_{k}$ polynomials in these variables; then the total degree of each $f_{k}\left(\sigma_{j}\left(X_{1}, \ldots, X_{n}\right): j=1, \ldots, n\right)$ is $k$.

Hence, using the polynomial expressions $f_{k}\left(\sigma_{j}: j\right)$, we can define the polynomial

$$
f\left(X_{0}, X_{1}, \ldots, X_{n}\right)=X_{0}^{\varepsilon}+\sum_{k=1}^{\varepsilon} f_{k}\left(\sigma_{1}, \ldots, \sigma_{k}\right) X_{0}^{\varepsilon-k}
$$

Clearly, $f\left(X_{0}, X_{1}, \ldots, X_{n}\right)$ is a polynomial of total degree $\varepsilon$, and, substituting $X_{i}=x_{i}, i=1, \ldots, n$ for which $s_{x_{1}, \ldots, x_{n}}$ contains an undetermined direction, 
yields the polynomial $f\left(X_{0}, x_{1}, \ldots, x_{n}\right)$ that splits completely into $\varepsilon$ linear factors. Also, since $f$ contains the term $X_{0}^{\varepsilon}$, the point $(1,0,0, \ldots, 0)$ is not a point of the hypersurface.

Suppose now that $f=\prod_{i} f_{i}$, where the polynomials $f_{i}\left(X_{1}, \ldots, X_{n}\right)$ are irreducible of degree $\varepsilon_{i}, \sum_{i} \varepsilon_{i}=\varepsilon$. Then each factor inherits the properties that (i) whenever the subspace $s_{x_{1}, x_{2}, \ldots, x_{n}} \subset H_{\infty}$ of dimension $n-2$ contains an undetermined direction, then $f_{i}\left(X_{0}, x_{1}, x_{2}, \ldots, x_{n}\right)$ splits into $\varepsilon_{i}$ linear factors; and (ii) $(1,0, \ldots, 0)$ is not a point of $f_{i}$. So from now on we will think of $f$ as an irreducible polynomial satisfying (i) and (ii).

$f\left(X_{0}, X_{1}, \ldots, X_{n}\right)=0$ is an algebraic hypersurface in the dual space $\mathrm{PG}(n, q)$. Our aim is to prove that it splits into $\varepsilon$ hyperplanes, or (equivalently) that it contains a linear factor (i.e. a hyperplane; then we can decrease $\varepsilon$ by one, etc.). Therefore, we state and prove a series of technical lemmas.

defhyperpl Lemma 7 Let $T \neq H_{\infty}$ be a deficient hyperplane through $\alpha=\left(\alpha_{0}, \alpha_{1}, \ldots, \alpha_{n}\right) \in$ $N$ (so $T$ contains less than $q^{n-2}$ points of $U$ ). Then in the dual space $\operatorname{PG}(n, q)$, $T$ corresponds to an intersection point $t$ of $f$ and the hyperplane $\left[\alpha_{0}, \alpha_{1}, \ldots, \alpha_{n}\right]$.

Proof. If $T=\left[x_{0}, x_{2}, \ldots, x_{n}\right]$ is a deficient hyperplane, then $x_{0}$ is a solution of the equation $f\left(X_{0}, x_{1}, x_{2}, \ldots, x_{n}\right)=0$, hence, in the dual space $\operatorname{PG}(n, q)$, $t=\left(x_{0}, x_{1}, \ldots, x_{n}\right)$ is a point of $f$. If $T$ contains $\alpha=\left(\alpha_{0}, \alpha_{1}, \ldots, \alpha_{n}\right) \in N$, then $t$ is contained in the hyperplane $\left[\alpha_{0}, \alpha_{1}, \alpha_{2}, \ldots, \alpha_{n}\right]$.

Lemma 8 Let $(\alpha) \in N$ be a non-determined direction. Then in the dual space $\operatorname{PG}(n, q)$ the intersection of the hyperplane $[\alpha]$ and $f$ is precisely the union of $\varepsilon$ different subspaces of dimension $n-2$.

Proof. First notice that

If $\left(0, \alpha_{1}, \alpha_{2}, \ldots, \alpha_{n}\right) \in H_{\infty}=[1,0, \ldots, 0]$ is an undetermined direction, then for all the subspaces $s_{x_{1}, x_{2}, \ldots, x_{n}} \subset H_{\infty}$ of dimension $n-2$ through $\left(0, \alpha_{1}, \alpha_{2}, \alpha_{3}, \ldots, \alpha_{n}\right)$ the polynomial $f\left(X_{0}, x_{1}, x_{2}, \ldots, x_{n}\right)$ has precisely $\varepsilon$ roots, counted with multiplicity.

translates to

In the hyperplane $\left[0, \alpha_{1}, \alpha_{2}, \ldots, \alpha_{n}\right] \ni(1,0, \ldots, 0)$, all the lines through $(1,0, \ldots, 0)$ intersect the surface $f\left(X_{0}, x_{1}, x_{2}, \ldots, x_{n}\right)=0$ in precisely $\varepsilon$ points, counted with intersection multiplicity.

Define $\bar{f}$ as the surface of degree $\bar{\varepsilon} \leq \varepsilon$, which is the intersection of $f$ and the hyperplane $\left[0, \alpha_{1}, \alpha_{2}, \ldots, \alpha_{n}\right]$. We know that all the lines through $(1,0, \ldots, 0)$ intersect $\bar{f}$ in precisely $\varepsilon$ points (counted with intersection multiplicity). So if $\bar{f}=\prod_{i} \bar{f}_{i}$, where $\bar{f}_{i}$ is irreducible of degree $\bar{\varepsilon}_{i}$ and $\sum_{i} \bar{\varepsilon}_{i}=\bar{\varepsilon}$, then we have that all the lines through $(1,0, \ldots, 0)$ intersect $\bar{f}_{i}$ in precisely $\bar{\varepsilon}_{i}$ points (counted with intersection multiplicity).

By Corollary 6 we know that there are precisely $\varepsilon$ different affine lines through the non-determined direction $(\alpha)$ not containing any point of $U$. In the dual space $\operatorname{PG}(n, q)$ these lines correspond to $\varepsilon$ different subspaces of dimension $n-2$ contained in the hyperplane $[\alpha]$. The deficient hyperplanes through these $\varepsilon$ original lines correspond to the points of the subspaces in the dual. Hence by 
Lemma 7 , all points of these subspaces are in $f$, which means that in $[\alpha]$ there are $\varepsilon$ different subspaces of dimension $n-2$ totally contained in $f$.

Now we prove a lemma, which is interesting for its own sake as well.

tanlemma Lemma 9 Let $f\left(X_{0}, \ldots, X_{n}\right)$ be a homogeneous polynomial of degree $d<q$. Suppose that there are $n-1$ independent concurrent lines $\ell_{1}, \ldots, \ell_{n-1}$ through the point $P$ in $\operatorname{PG}(n, q)$ totally contained in the hypersurface $f=0$. Then the hyperplane spanned by $\ell_{1}, \ldots, \ell_{n-1}$ is a tangent hyperplane of $f$.

Proof. Without loss of generality. let $P=(1,0,0, \ldots, 0)$ and $\ell_{i}$ be the "axis" $\langle P,(\stackrel{0}{1} \stackrel{1}{0}, 0, \ldots, 0, \stackrel{i}{1}, 0, \ldots, \stackrel{n}{0})\rangle, i=1, \ldots, n-1$. We want to prove that the hyperplane $x_{n}=0$, i.e. $[0, \ldots, 0,1]$ is tangent to $f$ at $P$.

Firstly, observe that $\partial_{X_{0}} f(P)=0$ as $f$ has no term of type $X_{0}^{d}$ since $f(P)=$ 0 .

Now we prove that $\partial_{X_{i}} f(P)=0$ for all $i=1, \ldots, n-1$. As $f$ vanishes on $\ell_{i}$ we have $f\left(s X_{i}, 0, \ldots, 0, X_{i}, 0, \ldots, 0\right)=0$ for all substitutions to $s$ and $X_{i}$. As $f\left(s X_{i}, 0, \ldots, 0, X_{i}, 0, \ldots, 0\right)=X_{i}^{d} f_{0}(s)$ for some $f_{0}$ with $\operatorname{deg} f_{0} \leq d<q$, we have $f_{0} \equiv 0$. In particular, $f_{0}$ has no term of degree $d-1$, so $f$ has no term of type $X_{0}^{d-1} X_{i}$. Hence $\partial_{X_{i}} f(1,0,0, \ldots, 0)=0$.

Corollary 10 Let $f\left(X_{0}, \ldots, X_{n}\right)$ be a homogeneous polynomial of degree $d<q$. Suppose that in $\operatorname{PG}(n, q)$ the intersection of a hyperplane $H$ and the hypersurface $f=0$ contains two complete subspaces of dimension $n-2$. Then $H$ is a tangent hyperplane of $f$.

Proof. Choose a point $P$ in the intersection of the two subspaces of dimension $n-2$, the lines $\ell_{1}, \ldots, \ell_{n-2}$ through $P$ in one of the subspaces and $\ell_{n-1}$ through $P$ in the other such that $\ell_{1}, \ldots, \ell_{n-1}$ be independent and apply Lemma 9 .

tangent Corollary 11 If $(\alpha)=\left(0, \alpha_{1}, \alpha_{2}, \ldots, \alpha_{n}\right) \in N \subset H_{\infty}$ is a non-determined direction, then (in the dual space) the hyperplane $[\alpha]$ is a tangent hyperplane of f. Note that $[\alpha]$ contains $(1,0, \ldots, 0)$.

Theorem 12 Let $n \geq 4$. Let $U \subset A G(n, q) \subset P G(n, q),|U|=q^{n-1}-2$. Let $D \subseteq H_{\infty}$ be the set of directions determined by $U$ and put $N=H_{\infty} \backslash D$ the set of non-determined directions. Then $U$ can be extended to a set $\bar{U} \supseteq U$, $|\bar{U}|=q^{n-1}$ determining the same directions only, or $|N| \leq\left\lfloor\frac{q+1}{2}\right\rfloor$, or each point of $N$ corresponds with a tangent plane to a hyperbolic quadric $\mathrm{Q}^{+}(3, q)$ in $\operatorname{PG}(3, q)$.

Proof. Let $n \geq 4$. The hypersurface $f=0$ is a quadric in the projective space $\operatorname{PG}(n, q)$. We will investigate the tangent hyperplanes through the point $(1,0, \ldots, 0)$ that meet $f=0$ in exactly two $(n-2)$-dimensional subspaces. If the quadric $f=0$ contains $(n-2)$-dimensional subspaces, then it must be singular, since $\lfloor(n-1) / 2\rfloor$ is an upper bound for the dimension of the generators. If $f=0$ contains 2 hyperplanes, then $f=0$ is the product of two linear factors, counted with multiplicity. But then the set $U$ can be extended. Hence, if we suppose that the set $U$ cannot be extended, the quadric $f=0$ contains $(n-2)$-dimensional subspaces, so it is a cone with vertex an $(n-3)$-dimensional subspace and base 
a conic, or it is a cone with vertex an $(n-4)$-dimensional subspace and base a hyperbolic quadric. Denote in both cases the vertex by $V$.

Firstly suppose that $f=0$ has an $(n-3)$-dimensional subspace as vertex. A tangent hyperplane $[\alpha]$ through $(1,0, \ldots, 0)$ containing two $(n-2)$-dimensional subspaces must meet the base conic in two points. When $q$ is odd, there are $\frac{q+1}{2}$, respectively $\frac{q-1}{2}$ such tangent hyperplanes, depending on whether the vertex $V$ is projected from the point $(1,0, \ldots, 0)$ onto an internal point, respectively, an external point of the base conic. When $q$ is even, there are $\frac{q}{2}$ such tangent hyperplanes.

Secondly suppose that $f=0$ has an $(n-4)$-dimensional subspace as vertex. Now a tangent hyperplane $[\alpha]$ through $(1,0, \ldots, 0)$ will meet the base quadric in two lines, i.e. a tangent plane to this hyperbolic quadric. Hence, there are exactly $q^{2}+2 q+1$ such tangent hyperplanes through the point $(1,0, \ldots, 0)$.

extend3 Theorem 13 Let $U \subset A G(3, q) \subset P G(3, q),|U|=q^{2}-\varepsilon$. Let $D \subseteq H_{\infty}$ be the set of directions determined by $U$ and put $N=H_{\infty} \backslash D$ the set of non-determined directions. Then $U$ can be extended to a set $\bar{U} \supseteq U,|\bar{U}|=q^{2}$ determining the same directions only, or $N$ is contained in a curve of $H_{\infty}$, of degree $\varepsilon(\varepsilon-1)^{2}$.

Proof. Our aim is to give a description of the tangent hyperplanes of $f$ passing through $(1,0,0,0)$. It is known (see [6], chapter 6 ) that there exists an algebraic curve $f^{*}=0$ in the dual space of $f$ (i.e. now in the original space as $f$ lives in its dual space), such that the points of $f^{*}$ correspond to tangent hyperplanes of $f$ and $\operatorname{deg} f^{*} \leq(\operatorname{deg} f)(\operatorname{deg} f-1)^{2}=\varepsilon(\varepsilon-1)^{2}$. Finally, the tangent hyperplanes passing through $(1,0,0,0)$ are contained in the intersection of this $f^{*}=0$ with the hyperplane $[1,0,0,0]=H_{\infty}$. This proves the theorem.

extend Theorem 14 Let $U \subset A G(n, q) \subset P G(n, q),|U|=q^{n-1}-\varepsilon$. Let $D \subseteq H_{\infty}$ be the set of directions determined by $U$ and put $N=H_{\infty} \backslash D$ the set of nondetermined directions. Then $U$ can be extended to a set $\bar{U} \supseteq U,|\bar{U}|=q^{n-1}$ determining the same directions only, or $N$ is contained in a hypersurface of $H_{\infty}$, of degree $\varepsilon(\varepsilon-1)^{n-1}$.

Proof. Our aim is to give a description of the tangent hyperplanes of $f$ passing through $(1,0, \ldots, 0)$. It is known (see [6], chapter 6$)$ that there exists an algebraic hypersurface $f^{*}=0$ in the dual space of $f$ (i.e. now in the original space as $f$ lives in its dual space), such that the points of $f^{*}$ correspond to tangent hyperplanes of $f$ and $\operatorname{deg} f^{*} \leq(\operatorname{deg} f)(\operatorname{deg} f-1)^{n-1}=\varepsilon(\varepsilon-1)^{n-1}$. Finally, the tangent hyperplanes passing through $(1,0, \ldots, 0)$ are contained in the intersection of this $f^{*}=0$ with the hyperplane $[1,0, \ldots, 0]=H_{\infty}$. This proves the theorem.

\section{Applications}

A (finite) generalized quadrangle (GQ) is an incidence structure $\mathcal{S}=(\mathcal{P}, \mathcal{B}, \mathrm{I})$ in which $\mathcal{P}$ and $\mathcal{B}$ are disjoint non-empty sets of objects called points and lines (respectively), and for which $I \subseteq(\mathcal{P} \times \mathcal{B}) \cup(\mathcal{B} \times \mathcal{P})$ is a symmetric point-line incidence relation satisfying the following axioms: 
(i) Each point is incident with $1+t$ lines $(t \geqslant 1)$ and two distinct points are incident with at most one line.

(ii) Each line is incident with $1+s$ points $(s \geqslant 1)$ and two distinct lines are incident with at most one point.

(iii) If $x$ is a point and $L$ is a line not incident with $x$, then there is a unique pair $(y, M) \in \mathcal{P} \times \mathcal{B}$ for which $x$ I $M$ I $y$ I $L$.

The integers $s$ and $t$ are the parameters of the GQ and $\mathcal{S}$ is said to have order $(s, t)$. If $s=t$, then $\mathcal{S}$ is said to have order $s$. If $\mathcal{S}$ has order $(s, t)$, then $|\mathcal{P}|=(s+1)(s t+1)$ and $|\mathcal{B}|=(t+1)(s t+1)$. (see e.g. [4]). The dual $\mathcal{S}^{D}$ of a GQ $\mathcal{S}=(\mathcal{P}, \mathcal{B}, \mathrm{I})$ is the incidence structure $(\mathcal{B}, \mathcal{P}, \mathrm{I})$. It is again a GQ.

We will introduce three classes of examples of GQs. Therefore we first need the definition of ovals, respectively ovoids in projective planes, respectively spaces.

An oval of $\operatorname{PG}(2, q)$ is a set of $q+1$ points $\mathcal{O}$, such that no three points of $\mathcal{O}$ are collinear. When $q$ is odd, it is known that all ovals of $\mathrm{PG}(2, q)$ are conics. When $q$ is even, several other examples and infinite families are known, see e.g. [3].

An ovoid of $\mathrm{PG}(3, q)$ is a set of $q^{2}+1$ points $\mathcal{O}$ such that no three points of $\mathcal{O}$ are collinear. When $q$ is odd, it is known that all ovoids of $\operatorname{PG}(3, q)$ are elliptic quadrics. When $q$ is even, one other infinite class of examples are known, i.e. the so-called Tits-ovoids [3].

Suppose that $\mathcal{O}$ is an oval of $\mathrm{PG}(2, q)$, then a line of $\mathrm{PG}(2, q)$ either intersects $\mathcal{O}$ in 0 points, 2 points or 1 points, in the latter case the line is called a tangent line. Suppose that $\mathcal{O}$ is an ovoid of $\mathrm{PG}(3, q)$, then a plane of $\mathrm{PG}(3, q)$ either intersects $\mathcal{O}$ in an oval, or in exactly one point. In the latter case, the plane is called a tangent plane.

Let now $n \in\{2,3\}$, and suppose that $\mathcal{O}$ is an oval of $\mathrm{PG}(2, q)$ when $n=2$ or $\mathcal{O}$ is an ovoid of $\operatorname{PG}(3, q)$ when $n=3$. Embed $\operatorname{PG}(n, q)$ as a hyperplane in $\mathrm{PG}(n+1, q)$. We denote this hyperplane with $\pi_{\infty}$. We define the following incidence structure, denoted as $T_{n}(\mathcal{O})$.

Define points as

(i) the points of $\mathrm{PG}(n+1, q) \backslash \mathrm{PG}(n, q)$,

(ii) the hyperplanes $\pi$ of $\operatorname{PG}(n+1, q)$ for which $|\pi \cap \mathcal{O}|=1$, and

(iii) one new symbol $(\infty)$.

Lines are defined as

(a) the lines of $\mathrm{PG}(n+1, q)$ which are not contained in $\mathrm{PG}(n, q)$ and meet $\mathcal{O}$ (necessarily in a unique point), and

(b) the points of $\mathcal{O}$.

Incidence between points of type (i) and (ii) and lines of type (a) and (b) is the inherited incidence of $\mathrm{PG}(n+1, q)$. In addition, the point $(\infty)$ is incident with no line of type (a) and with all lines of type (b).

It is straightforward to show that these incidence structure is a GQ of order $q$ when $n=2$ and of order $\left(q, q^{2}\right)$ when $n=3$. 
When $q$ is even, it is well known that all tangent lines to an oval of $\mathrm{PG}(2, q)$ are the lines through a common point. This common point is called the nucleus. Adding the nucleus $n$ to the oval $\mathcal{O}$, one obtains a set of $q+2$ points, such that no line contains more than 2 points, and such that no line is tangent. Such a set is called a hyperoval. Clearly, removing exactly one point from a hyperoval, yields an oval.

Consider a hyperoval $\mathcal{H}$ of $\mathrm{PG}(2, q), q$ even. Embed $\mathrm{PG}(2, q)$ as a hyperplane in $\mathrm{PG}(3, q)$. We introduce now the following incidence structure, denoted as $T_{2}^{*}(\mathcal{H})$. Define points as the set of points of $\mathrm{PG}(3, q) \backslash \mathrm{PG}(2, q)$, define the lines as the lines of $\operatorname{PG}(3, q)$ which are not contained in $\operatorname{PG}(2, q)$ and meet $\mathcal{H}$ (necessarily) in a unique point, and define incidence as the inherited incidence of $\operatorname{PG}(3, q)$. It is straightforward to show that this incidence structure is a GQ of order $(q-1, q+1)$.

An ovoid of a GQ $\mathcal{S}$ is a set $\mathcal{O}$ of points of $\mathcal{S}$ such that every line is incident with exactly one point of the ovoid. An ovoid of a GQ of order $(s, t)$ has necessarily size $1+$ st. A partial ovoid of a GQ is a set $\mathcal{K}$ of points such that every line contains at most one point of $\mathcal{K}$. An partial ovoid $\mathcal{K}$ is called maximal if and only if $\mathcal{K} \cup\{p\}$ is not an partial ovoid for any point $p \in \mathcal{P} \backslash \mathcal{K}$, in other words, if $\mathcal{K}$ cannot be extended. It is clear that any arc of a GQ of order $(s, t)$ contains $1+s t-\rho$ points, $\rho \geq 0$, with $\rho=0$ if and only if $\mathcal{K}$ is an ovoid.

We are interested in ovoids (if existing) and (maximal) partial ovoids of the above introduced GQs $T_{2}(\mathcal{O}), T_{3}(\mathcal{O})$ and $T_{2}^{*}(\mathcal{H})$. It is easy to construct an ovoid of the GQs $T_{2}(\mathcal{O})$ and $T_{2}^{*}(\mathcal{H})$. Consider any plane $\pi$ of $\operatorname{PG}(3, q)$ not meeting $\mathcal{O}$. Then the set of $q^{2}$ points $\pi \backslash \pi_{\infty}$ together with the point $\infty$ constitutes an ovoid of $T_{2}(\mathcal{O})$. Considering $T_{2}^{*}(\mathcal{H})$, it is possible to construct in the same way an ovoid: consider the $q^{2}$ points not in $\pi_{\infty}$ of any plane $\pi$ not meeting $\mathcal{H}$. This set is an ovoid of $T_{2}^{*}(\mathcal{H})$.

The following theorems are from [4].

Theorem 15 The $G Q T_{3}(\mathcal{O})$ has no ovoids

On partial ovoids of GQs, we mention the following theorem.

Theorem 16 Consider a GQ of order $(s, t)$. Any partial ovoid of size $(s t-\rho)$, with $0 \leq \rho<t / s$ is contained in a uniquely defined ovoid.

We first consider the GQs $T_{n}(\mathcal{O}), n \in\{2,3\}$. An ovoid of such a GQ has size $q^{n}+1$. These GQs are so-called translation generalized quadrangles. The point $\infty$ plays a special role with relation to the symmetry group. It is known (see e.g. [7]) that such a GQ contains always a line consisting completely of translation points, i.e. points that can play the role of $\infty$ in their description. Hence, If $\mathcal{B}$ is an ovoid of such a GQ, we can always choose $\infty \in \mathcal{B}$. The set $\mathcal{B} \backslash\{\infty\}$ is then necessarily a set of $q^{n}$ points of type (i) of the GQ, such that no two points determine a line of the GQ. Hence, with an ovoid of such a GQ corresponds a set of $q^{n}$ points, not determining a point of $\mathcal{O}$ at infinity, and with a partial ovoid corresponds a set of $q^{n}-\epsilon$ points not determining a point of $\mathcal{O}$ at infinity. Since the point set of the GQ $T_{2}^{*}(\mathcal{H})$ consists only of affine points, it is also clear that a partial ovoid of $T_{2}(\mathcal{H})$ corresponds with a set of $q^{2}-\epsilon$ affine points not determining a point of $\mathcal{H}$ at infinity.

An argument of [2] shows in fact the a set $U$ of affine points in $\operatorname{AG}(n, q)$, $n \in\{3,4\},|U|=q^{n}-1$, not determining the set $\mathcal{O}$ at infinity (where $\mathcal{O}$ is an 
oval of $\mathrm{PG}(2, q)$, a hyperoval of $\mathrm{PG}(2, q)$, or an ovoid of $\mathrm{PG}(3, q)$ respectively, can always be extended to a set $\bar{U},|\bar{U}|=q^{n}$, not determining the same set $\mathcal{O}$ at infinity. In other words, a partial ovoid of $T_{n}(\mathcal{O})$, of size $q^{n}, T_{2}^{*}(\mathcal{H})$, of size $q^{2}-1$ respectively, can always be extended.

Using Theorem 14, we can now formulate the following corollaries.

cort2o Corollary 17 A partial ovoid of $T_{2}(\mathcal{O}), \mathcal{O}$ a non-classical oval of $\mathrm{PG}(2, q)$, of size $q^{2}-1$ can always be extended.

Proof. A partial ovoid of $T_{2}(\mathcal{O})$ of size $q^{2}-1$ is gives rise to a set $U$ of size $q^{2}-2$ of affine points in $\operatorname{AG}(3, q)$, not determining the set $\mathcal{O}$ at infinity. If the partial ovoid is maximal, then any extension $\bar{U}$ of the set $U$ will determine at least one direction of $\mathcal{O}$. Hence, by Theorem 13, the set of non-determined directions, so in particular the set $\mathcal{O}$, is contained in an algebraic curve of $\mathrm{PG}(2, q)$ of degree 2 , a contradiction, since $\mathcal{O}$ is a non-classical oval.

Notice that this corollary is an improvement of Theorem 16 for the mentioned GQ. The following corollary is proved using Theorem 12, but it follows also from Theorem 16

cort3o Corollary 18 A partial ovoid of $T_{3}(\mathcal{O})$ of size $q^{3}-1, \mathcal{O}$ an ovoid of $\mathrm{PG}(3, q)$ can always be extended.

Proof. A partial ovoid of $T_{2}(\mathcal{O})$ of size $q^{2}-1$ is gives rise to a set $U$ of size $q^{2}-2$ of affine points in $\operatorname{AG}(3, q)$, not determining the set $\mathcal{O}$ at infinity. If the partial ovoid is maximal, then any extension $\bar{U}$ of the set $U$ will determine at least one direction of $\mathcal{O}$. The non-determined directions are exactly the points of the ovoid $\mathcal{O}$. Hence, by $\mathrm{T}$ theorem 12 , there are either less than $\frac{q+1}{2}$ non-determined directions, which is not possible since $|\mathcal{O}|=q^{2}+1$, or the set of non-determined directions is contained in a hyperbolic quadric $\mathrm{Q}^{+}(3, q)$, a contradiction, since no ovoid of $\operatorname{PG}(3, q)$ is contained in a hyperbolic quadric. Hence, the partial ovoid cannot be maximal.

Now we remark that when $\mathcal{O}$ is a classical oval of $\operatorname{PG}(2, q)$, i.e., a conic, and $\mathcal{K}$ is a partial ovoid of size $q^{2}-1$ of $T_{2}(\mathcal{O})$, then, by Theorem 14 , the set of undetermined directions is contained in an algebraic curve of degree 2 , which is exactly the conic $\mathcal{O}$. Hence, Theorem 12 cannot exclude maximal partial ovoids of $T_{2}(\mathcal{O})$, of size $q^{2}-1$ when $\mathcal{O}$ is a conic. However, these objects are excluded when $q=p^{h}, h>1$, in [2], but surprisingly, there are examples of such objects for $q \in\{3,5,7,11\}$.

Finally, for the GQ $T_{2}(\mathcal{H})$, the following corollary can be proved in exactly the same way as Corollary 17. In principle, techniques as used in [2] could lead to the same result, provided that Theorem 2 can be translated, at least for $n=3$, to $q$ even.

cort2h Corollary 19 A partial ovoid of $T_{3}(\mathcal{H}), \mathcal{H}$ a hyperoval of $\mathrm{PG}(2, q), q$ even, of size $q^{2}-2$ can always be extended.

\section{References}

Ball:2011 [1] S. Ball. The polynomial method in Galois geometries. In Current research topics in Galois geometry, chapter 5, pages 101-126. Nova Sci. Publ., to appear, New York. 
DBG2008 [2] J. De Beule and A. Gács. Complete arcs on the parabolic quadric Q $(4, q)$. Finite Fields Appl., 14(1):14-21, 2008.

DCD:2011 [3] F. De Clerck and N. Durante. Constructions and characterizations of classical sets in $\mathrm{PG}(n, q)$. In Current research topics in Galois geometry, chapter 1, pages 1-32. Nova Sci. Publ., to appear, New York.

PT:2009 [4] S. E. Payne and J. A. Thas. Finite generalized quadrangles. EMS Series of Lectures in Mathematics. European Mathematical Society (EMS), Zürich, second edition, 2009.

Szonyi1996 [5] T. Szőnyi. On the number of directions determined by a set of points in an affine Galois plane. J. Combin. Theory Ser. A, 74(1):141-146, 1996.

Tevelev2005 [6] E. A. Tevelev. Projective duality and homogeneous spaces, volume 133 of Encyclopaedia of Mathematical Sciences. Springer-Verlag, Berlin, 2005. Invariant Theory and Algebraic Transformation Groups, IV.

Thas:2004 [7] K. Thas. Symmetry in finite generalized quadrangles. Frontiers in Mathematics. Birkhäuser Verlag, Basel, 2004. 